\title{
Les sources protéiques dans les aliments pour les poissons d'élevage
}

Françoise Médale

Sadasivam Kaushik

Inra

UMR 1067 Nutrition, aquaculture

et génomique

Pôle hydrobiologie

Ifremer

Université de Bordeaux

64310 Saint-Pée-sur-Nivelle

France

$<$ medale@st-e.inra.fr>

$<$ kaushik@st-pee.inra.fr>

\begin{abstract}
Résumé
Les poissons nécessitent un apport protéique alimentaire élevé (30 à $55 \%$ selon les espèces) par rapport aux autres animaux d'élevage et un apport en acides aminés répondant précisément à leur besoin. La farine de poisson est la meilleure source protéique pour les aliments piscicoles en raison de sa teneur en protéines (64 à $72 \%$ ), de son profil en acides aminés indispensables qui couvre les besoins des poissons et de l'absence de facteurs antinutritionnels. Cependant, il est devenu nécessaire de lui trouver des substituts afin de limiter la dépendance de l'aquaculture aux produits de la pêche et d'épargner les ressources marines. Les premiers essais ont été réalisés avec des coproduits de la production animale (farine de viande, de sang, d'os, de plume) qui sont maintenant interdits en Europe. Les substituts potentiels les plus étudiés sont les sources protéiques végétales. Leurs inconvénients majeurs sont leur faible taux protéique, leur profil en acides aminés et la présence de facteurs antinutritionnels. Les extraits protéiques végétaux, tels que les glutens ou les concentrés protéiques, présentent des taux protéiques plus élevés et des facteurs antinutritionnels en moindre quantité, mais leur prix, à l'heure actuelle très élevé, limite leur emploi. Les nombreuses études conduites au cours des 30 dernières années ont permis de cerner l'intérêt et les limites des différents substituts disponibles. L'utilisation de mélanges de sources protéiques végétales et l'ajout de certains acides animés indispensables rendent possible le remplacement de 75 à $95 \%$ de la farine de poisson chez la plupart des espèces de poissons. Cependant, des efforts de recherche restent à réaliser pour identifier les limites biologiques au remplacement total, les modes d'action des facteurs antinutritionnels, et pour ajuster l'apport en micronutriments (vitamines et minéraux) afin d'optimiser les régimes à base de protéines végétales pour les différentes espèces de poissons d'élevage.
\end{abstract}

Mots clés : acide aminé ; alimentation des poissons ; facteur antinutritionnel ; farine de poisson; protéine végétale.

Thèmes : alimentation, consommation, nutrition ; pêche et aquaculture ; productions animales ; recherche scientifique et agronomique.

\section{Abstract \\ Protein sources in feed for farmed fish}

Fish require diets containing 30 to $55 \%$ of crude protein and an amino acid supply precisely adapted to meeting the needs for optimal growth. Fish meal is an ideal protein source for aquafeed because it has a high protein level (65\% to $72 \%)$ with an ideal proportion of all ten indispensable amino acids that meet the requirements of all fish species. However, finding alternatives to fish meal has become an absolute necessity in order to reduce the reliance of aquaculture on marine ingredients. Animal by-product meals (meat meal, bone meal, blood meal, poultry by-product meal, hydrolysed feather meal) are used as alternative protein sources in aquafeed in extra-European countries. Since the use of animal protein in aquafeed is forbidden in Europe (except non ruminant blood meal), more attention has been given to the use of plant protein sources which are available in large amounts on the market. The limitations as regards their incorporation in diets for fish are related to the level of protein, the amino acid profiles and the presence of anti-nutritional factors (ANF). Protein extracts from oilseeds and cereals are higher in protein than meals and pulses and lower in ANF but currently more expensive, thus limiting their use in aquafeed. Numerous studies undertaken with different fish species have led to improved knowledge on the potential of various plant ingredients. Blends of plant protein sources duly supplemented with the required indis- 
L

es protéines représentent de 30 à plus de $50 \%$ de la ration alimentaire des poissons. La farine de poisson a été choisie comme principale source protéique en raison de sa teneur élevée en protéines et de sa composition en acides aminés répondant aux besoins des poissons. Cependant, dès les années 1970, des recherches ont été entreprises pour lui trouver des substituts afin de diversifier les sources protéiques des aliments piscicoles et éviter ainsi que la production de poissons soit dépendante d'une matière première largement majoritaire. En plus des efforts visant à réduire le taux protéique des régimes (voir chapitre concernant les lipides), de nombreux essais ont été conduits pour évaluer l'intérêt et les limites de l'incorporation de coproduits d'abattoir (farines de plumes, de viande, d'os, de sang), de l'industrie laitière (bactéries, lactosérum) ou des brasseries (drèches de brasserie, malt, etc.). Du fait de leur très grande disponibilité sur le marché, les sources protéiques végétales ont aussi fait l'objet d'études. Au cours des dernières années, l'essor soutenu de l'aquaculture $(+8,5 \%$ ) an ; FAO, 2006) a provoqué une demande accrue d'aliments composés, mais la production mondiale de farine de poisson est restée stable (Shepherd et al., 2005; Tacon et Metian, 2008). Cette situation et l'impérative nécessité de protéger les stocks de ressources naturelles marines ont accentué l'intérêt porté à la substitution de la farine de poisson par d'autres sources de protéines. Du fait de l'interdiction d'utiliser des produits d'origine animale dans l'alimentation des poissons en Europe, les recherches sur les sources protéiques végétales se sont intensifiées. Les ingrédients alternatifs ne doivent pas augmenter le coût des aliments qui représente la majeure partie du coût de la production piscicole. Ils doivent permettre pensable amino acids can replace 75 to $95 \%$ of fish meal in almost all species, thus reducing the pressure of aquaculture on marine resources. Further research should focus on factors that can facilitate total replacement, on the origin of the adverse effects of ANF and on the dietary supply of micronutrients (minerals and vitamins) in order to improve the ormulation of totally plant-protein-based diets for fish.

Key words: amino acids; antinutritionnal factors; fish feeding; fish meal; plant proteins.

Subjects: animal productions; fishing and aquaculture; food, consumption, nutrition; scientific and agricultural research. une croissance optimale des poissons sans altérer leur bien-être et leur santé, avoir un impact minimal sur l'environnement et fournir des produits attractifs sur le plan sensoriel et nutritionnel. Cet article fait une synthèse des différentes sources protéiques potentiellement utilisables dans les aliments aquacoles en décrivant leur intérêt et leurs limites.

\section{Besoins en protéines et acides aminés des poissons}

Les protéines apportées par l'alimentation doivent permettre aux poissons de cou- vrir leurs besoins azotés pour la croissance à la fois en terme quantitatif et qualitatif. Le tableau 1 rassemble les valeurs des taux de protéines alimentaires moyens nécessaires pour la croissance de différentes espèces de poisson. Si ces valeurs sont plus élevées pour les poissons dits carnivores, comme les salmonidés ou les espèces marines (40 à $55 \%$ de la ration), on notera que mêmes des poissons réputés non carnivores comme la carpe, le poisson-chat américain et le tilapia requièrent au moins $30 \%$ de protéines dans leur ration alimentaire, ce qui est élevé en comparaison des autres animaux d'élevage (20\% maximum pour le poulet de chair). Cela s'explique par le fait que les poissons utilisent une partie des protéines alimentaires

\section{Tableau 1. Niveaux protéiques alimentaires nécessaires pour la croissance de quelques espèces de poissons.}

Table 1. Protein requirements for growth of some fish species.

\section{Espèce}

Saumon atlantique (Salmo salar)

Saumon coho (Oncorhynchus kisutch)

Truite arc-en-ciel (Oncorhynchus mykiss)

Truite fario (Salmo trutta)

Esturgeon (Acipenser baeri)

Bar (Dicentrarchus labrax)

Daurade (Sparus aurata)

Turbot (Psetta maxima)

Sériole (Seriola quinqueradiata)

Poisson-chat américain (Ictalurus punctatus)

Carpe commune (Cyprinus carpio)

Tilapia du Nil (Oreochromis niloticus)
Teneur en protéines de l'aliment (pourcentage de la matière sèche)
D'après NRC, 1993.
45
40
38-40
53
40
$45-50$
$40-45$
55
55

$32-36$

31-38

30 
pour la fourniture d'énergie. Malgré cette caractéristique, les poissons convertissent les protéines alimentaires en protéines corporelles de manière plus efficace que les autres animaux d'élevage, car leurs besoins énergétiques sont plus faibles. Animaux poïkilothermes (dont la température du corps est identique à celle du milieu), ils font l'économie des dépenses énergétiques pour la thermorégulation, premier poste de dépenses d'énergie chez les homéothermes.

Au-delà de la quantité de protéines, les sources protéiques de l'aliment doivent fournir des acides aminés en quantité adéquate pour permettre une utilisation optimale de l'apport protéique. Elles doivent contenir les acides aminés indispensables (AAI) pour couvrir les besoins des poissons.

Chez les poissons, comme chez d'autres animaux d'élevage, les mêmes dix acides aminés sont indispensables: arginine, histidine, isoleucine, leucine, lysine, méthionine, phénylalanine, thréonine, tryptophane et valine. Les besoins quantitatifs en AAI ne sont pas connus pour toutes les espèces. Néanmoins, des données existent pour les principales espèces d'intérêt aquacole (NRC, 1993 ; Tibaldi et Kaushik, 2005). En comparant les don- nées bibliographiques de composition en AAI de différents tissus d'un grand nombre d'espèces de poisson et leurs besoins en AAI, Mambrini et Kaushik (1995) ont constaté que la composition en AAI du corps entier était le meilleur reflet des besoins en AAI et que ces besoins étaient très conservés entre espèces. Lorsque l'apport alimentaire est déficient en un AAI, on observe une augmentation des excréta azotés provenant de la désamination oxydative des acides aminés, reflétant leur dégradation accrue (figure 1). Pour que les acides aminés puissent être utilisés pour la synthèse protéique, il est important que l'apport alimentaire soit équilibré entre AAI et AANI (acides aminés non indispensables). En nourrissant des truites avec des aliments contenant des acides aminés en quantités variables pour obtenir six rapports AAI/AANI différents, Green et al. (2002) ont trouvé qu'un rapport AAI/ AANI de 46/54 permettait une rétention azotée optimale. Ces valeurs sont similaires à celles recommandées pour les mammifères et les oiseaux.

Pour satisfaire ces besoins, il faut donc que les matières premières incorporées dans les aliments aquacoles répondent à des caractéristiques particulières en ter-

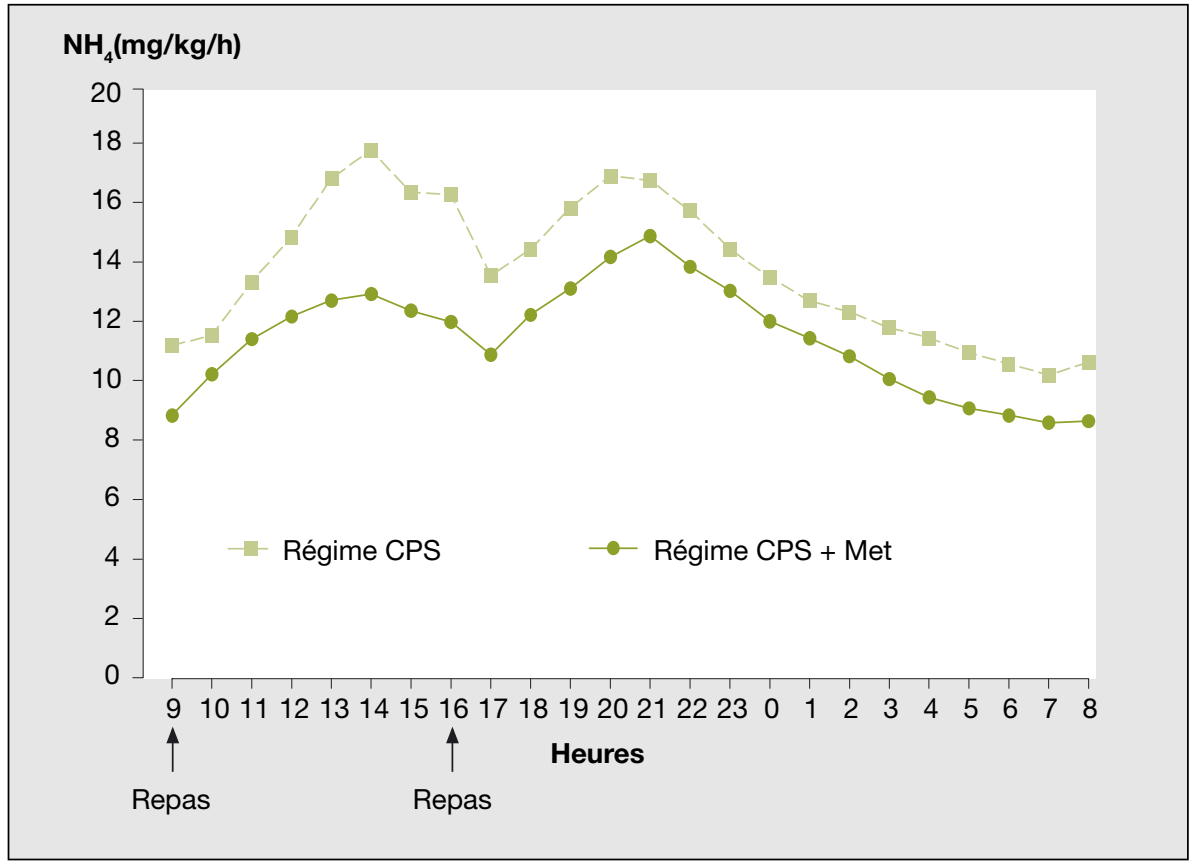

Figure 1.Excrétion d'azote ammoniacal de truites arc-en-ciel nourries avec un régime déficient en méthionine (régime CPS) ou supplémenté en méthionine (CPS + Met) pour couvrir les besoins ( $d$ 'après Médale et al., 1998).

Figure 1. Ammonia excretion in rainbow trout fed a diet deficient in methionine (diet CPS) or a diet with added coated crystalline Met (diet CPS + Met) (redrawn from Médale et al., 1998). mes de taux protéique, de composition et de disponibilité en acides aminés.

\section{Sources protéiques d'origine marine}

\section{Farines de poisson}

La majorité des farines de poisson sont produites à partir de poissons entiers, présentant un faible intérêt pour le marché de consommation humaine (petite taille, présence d'arêtes). La pêche minotière (spécifique pour la production de farines et huiles de poisson) concerne l'anchois du Pérou (Engraulis ringens), le merlan bleu (Micromesistius poutassou), l'anchois japonais (Engraulis japonicus) et certaines espèces de maquereaux, de sardines et de hareng (Shepherd et al., 2005). Le Pérou produit près d'un tiers de toutes les farines de poisson utilisées dans le monde (majoritairement de la farine d'anchois) ; les autres principaux pays producteurs sont le Chili, la Thailande, la Chine, les États-Unis, le Danemark, l'Islande et la Norvège. Au cours des 20 dernières années, la production a fluctué entre 5,5 et 7,2 millions de tonnes par an (Shepherd et al., 2005). Les plus fortes fluctuations sont liées à des événements climatiques tels qu'El Niño. En effet, les pêches minotières sont gérées par un système de quotas afin d'éviter la surpêche et de permettre le renouvellement des stocks et la durabilité de ces espèces.

Les procédés industriels de cuisson, de pressage, d'ajout de solubles et de séchage permettent de préserver la valeur biologique de la farine de poisson pour l'alimentation animale. Il faut quatre à cinq tonnes (selon la teneur en protéines corporelles des espèces) de poisson frais entier pour produire une tonne de farine de poisson. Les farines de poisson contiennent de 66 à $72 \%$ de protéines (selon l'espèce et le procédé de fabrication), de teneurs en AANI et AAI équilibrées. Leur composition en AAI correspond à l'apport nécessaire pour couvrir les besoins des différentes espèces de poissons (tableau 2). Elles peuvent aussi contenir jusqu'à $12 \%$ d'huile (selon l'espèce et le procédé de fabrication), riche en acides gras longs polyinsaturés (AGPI) de la série oméga 3, ainsi que des minéraux et des vitamines qui contribuent à couvrir les besoins des poissons. 


\section{Autres sources protéiques d'origine aquatique}

On ne connaît pas d'autres ingrédients d'origine marine disponibles en quantités suffisantes et ayant les qualités requises pour servir de substituts à la farine de poisson.

Le concentré de protéines solubles de poisson (CPSP) est obtenu, à partir de poissons broyés, par hydrolyse enzymatique des protéines. Le produit d'hydrolyse est séché par atomisation après avoir été délipidé ou non (CPSPG). Composé de peptides et d'acides aminés qui sont très facilement digérés, son ingestion se traduit par un apport massif d'acides aminés dont une partie est catabolisée. Son incorporation dans les aliments doit donc être limitée.

Parmi les autres produits aquatiques, le krill (petits crustacés des eaux froides) suscite l'intérêt, car sa composition en AAI est proche de celle des farines de poisson (tableau 2). Le remplacement total de la farine de poisson par de la farine de krill, sans altérer la croissance des animaux, a été réalisé expérimentalement chez la morue et la truite arc-en-ciel (Moren et al., 2007). Cependant, l'utilisation de ce produit est limitée en raison de sa forte concentration en fluor (1 000$6000 \mathrm{mg} / \mathrm{kg}$ ), le niveau maximum de fluor dans les aliments piscicoles étant fixé par une directive européenne de 1999 à $150 \mathrm{mg} / \mathrm{kg}$ aliment sec. Actuellement, la farine de krill est surtout employée, à des taux de 5 à $10 \%$, pour ses propriétés de stimulation de l'appétit dans les aliments à base de végétaux (Palti et al., 2006). Il en est de même pour les farines de crevettes (fabriquées à partir des céphalothorax) et de calmar. Ces trois produits servent donc plus d'additifs que de sources protéiques majeures.

Les quelques essais réalisés avec des algues n'ont pas eu le développement espéré. Un inconvénient majeur est la teneur en polysaccharides complexes, constituants des parois, que les animaux aquatiques ne peuvent pas digérer du fait de l'absence d'enzymes spécifiques et d'une flore digestive appropriée. Des essais avec des spirulines montrent la possibilité de remplacer $40 \%$ de la farine de poisson par cet ingrédient dans l'alimentation du tilapia (Olvera-Novoa et al., 1998). La nécessité de diversifier les sources protéiques pourrait stimuler l'intérêt pour ces matières premières actuellement peu utilisées dans les aliments aquacoles.

\section{Coproduits d'origine animale}

Dans les années 1970-1980, des essais de valorisation de coproduits d'origine animale ont connu un certain intérêt.

Les farines de viande, fabriquées à partir de déchets d'abattoir et de boucherie, contiennent 50 à $70 \%$ de protéines, mais la température de cuisson, qui doit toujours être élevée pour des raisons sanitaires, ne permet pas d'obtenir des protéines aussi digestibles que celles des farines de poisson. Les farines de viandes sont limitées en acides aminés soufrés (Met et Cys - tableau 2). Lorsqu'il s'agit de farine de viande et d'os, c'est le faible apport protéique joint à l'apport minéral élevé qui pose problème pour son emploi dans les aliments aquacoles.

Les coproduits de volailles, tels que la farine de déchets (viscères principalement), ou d'hydrolysats de plumes sont des ingrédients ayant une valeur biologique élevée, à condition d'avoir subi des traitements technologiques appropriés, et sont couramment utilisés dans divers pays du monde dans les aliments pour poissons ou crevettes.

\section{Tableau 2. Teneur en protéines brutes (pourcentage de la matière sèche) et en acides aminés indispensables $(\mathrm{g} / 16 \mathrm{~g}, \mathrm{~N})$ des farines de poisson et autres sources protéiques animales ${ }^{a}$ - comparaison avec la fourchette des besoins des différentes espèces de poissons d'élevage.}

Table 2. Crude protein level (percent of dry matter) and indispensable amino-acids ( $\mathrm{g} / 16 \mathrm{~g}, \mathrm{~N}$ ) of fishmeal and other animal protein sources $^{a}$ - comparison with the range of amino-acid requirements of farm fish species.

\begin{tabular}{|c|c|c|c|c|c|c|c|}
\hline $\begin{array}{l}\text { Acides aminés } \\
\text { indispensables }\end{array}$ & $\begin{array}{l}\text { Besoins } \\
\text { de différentes } \\
\text { espèces }\end{array}$ & $\begin{array}{c}\text { Farines } \\
\text { de poisson }\end{array}$ & $\begin{array}{l}\text { Farine } \\
\text { de krill }\end{array}$ & $\begin{array}{c}\text { Farine } \\
\text { de plume }\end{array}$ & $\begin{array}{c}\text { Farine } \\
\text { de viande }{ }^{c}\end{array}$ & $\begin{array}{c}\text { Farine } \\
\text { de viande et os }\end{array}$ & $\begin{array}{c}\text { Farine } \\
\text { de sang }^{d}\end{array}$ \\
\hline Protéines brutes (\%) & $30-55$ & $66-72$ & 60 & $80-85$ & $60-70$ & 50 & 84 \\
\hline$A R G$ & $4,1 \pm 0,7$ & 5,8 & 6,0 & 7,3 & 6,4 & 7,2 & 4,4 \\
\hline HIS & $1,6 \pm 0,2$ & 2,4 & 2,0 & 0,9 & 1,9 & 1,8 & 6,7 \\
\hline ILE & $2,3 \pm 0,3$ & 4,3 & 4,9 & 5,2 & 2,8 & 2,7 & 1,5 \\
\hline LEU & $3,2 \pm 0,3$ & 7,2 & 7,6 & 8,8 & 5,7 & 6,8 & 13,4 \\
\hline LYS & $4,6 \pm 0,2$ & 7,5 & 8,2 & 2,6 & 5,1 & 5,0 & 9,7 \\
\hline METCYS & $2,7 \pm 0,5$ & 3,7 & 4,8 & 5,0 & 2,1 & 2,2 & 1,6 \\
\hline PHETYR & $4,8 \pm 0,5$ & 7,0 & 8,7 & 8,7 & 5,4 & 5,4 & 10,9 \\
\hline THR & $2,5 \pm 0,8$ & 4,2 & 4,3 & 5,0 & 3,1 & 3,1 & 4,5 \\
\hline TRP & $0,6 \pm 0,2$ & 1,0 & 1,2 & 0,6 & 0,6 & 0,5 & 1,4 \\
\hline VAL & $2,9 \pm 0,3$ & 5,1 & 5,4 & 7,4 & 4,3 & 4,2 & 8,3 \\
\hline
\end{tabular}

a Besoins des poissons d'après NRC, 1993 ; composition des matières premières d'après le site FAO et d'après Watanabe (2002).

b ARG : arginine ; HIS : histidine ; ILE : isoleucine ; LEU : leucine ; LYS : Iysine ; METCYS : méthionine + cystéine ; PHETYR : phénylalanine + tyrosine ; THR : thréonine; TRP : tryptophane; VAL : valine.

${ }^{c}$ Emploi interdit en Europe dans les aliments piscicoles.

d Depuis 2006, les dérivés du sang d'animaux non ruminants sont de nouveau autorisés dans les aliments piscicoles, mais ils restent peu utilisés. 
La farine de sang, obtenue par déshydratation du sang d'abattoir ayant subi un séchage par micronisation, conduit à un produit de bonne qualité. Malgré sa teneur en protéines élevée (85\%), sa valeur biologique est limitée du fait de ses faibles concentrations en méthionine, isoleucine et arginine et du large excès de lysine (tableau 2). Elle présente des propriétés intéressantes de liant. Elle est utilisée dans de nombreux pays extraeuropéens. Son incorporation dans les aliments pour les poissons d'élevage a été interdite en Europe, en 2002, au même titre que celle des autres produits d'origine animale, suite à la crise de l'encéphalopathie spongiforme bovine (ESB). Bien que la farine de sang d'animaux non ruminants soit de nouveau autorisée comme ingrédient de l'aliment piscicole depuis 2006, son utilisation est actuellement très restreinte en France.

Chez la carpe, le poisson-chat africain et le tilapia, ces coproduits peuvent remplacer 50 à $75 \%$ de la farine de poisson sans modifier la croissance (Yang et al., 2004 ; Goda et al., 2007). Ils présentent aussi un intérêt pour les salmonidés et les poissons marins. Watanabe et al., (1998) ont montré, chez la truite arc-en-ciel, la possibilité de remplacer totalement la farine de poisson de l'aliment, sans altérer la croissance, en utilisant un mélange de différents coproduits d'origine animale et végétale.

\section{Sources protéiques végétales}

Les produits d'origine végétale utilisables comme sources de protéines sont très nombreux, mais aucun ne réunit tous les atouts de la farine de poisson, en termes de disponibilité, de composition et de prix. Les oléagineux sont disponibles sous forme de tourteaux (coproduits de l'extraction de l'huile) ou, pour le soja et le colza, sous forme de concentré protéique. Les céréales telles que le blé et le mais sont riches en amidon et pauvres en protéines (10 à $20 \%$ ). Ce sont donc les extraits protéiques issus du fractionnement des céréales qui peuvent être utilisés pour l'alimentation aquacole, mais leur prix limite leur intérêt. Pour l'instant, les coproduits de distillerie pour la production d'éthanol sont trop riches en fibres pour être utilisables mais pourraient devenir intéressants si les procédés de fractionnement s'améliorent. Certains protéagineux tels que le pois, le lupin et, dans une moindre mesure (car moins disponibles), les féveroles présentent aussi un intérêt.

\section{Composition des différentes sources protéiques végétales}

Le premier inconvénient des végétaux est leur faible teneur en protéines, à l'exception des extraits (gluten de blé et de maïs) ou des concentrés (soja, colza) protéiques (60 à $80 \%$ ). Cependant, le procédé d'extraction rend ces produits onéreux par rapport aux farines de protéagineux ou aux tourteaux. Le tableau 3 regroupe les principales caractéristiques des tourteaux et des protéagineux disponibles comme sources protéiques pour les aliments aquacoles. Les produits végétaux ont des compositions en acides aminés moins bien adaptées aux besoins des poissons (figure 2), notamment en raison de la faible teneur en méthionine et lysine de la majorité d'entre eux (tableau 3). Ils ont des teneurs variables en amidon qui leur confère un pouvoir liant élevé, mais qui, à l'état natif, est faiblement digéré par les salmonidés et les poissons marins. La plupart des produits végétaux contiennent des polysaccharides non amylacés (principaux composants des fibres) que les animaux aquatiques ne peuvent pas digérer du fait de l'absence d'enzymes spécifiques et d'une flore digestive appropriée. Ils réduisent l'efficacité alimentaire, semble-t-il par diminution de la digestibilité des graisses et des protéines, chez le saumon comme chez le poulet (Refstie et al., 1999; Amirkolaie et al., 2005; Krogdahl et al., 2005). Enfin, les produits végétaux contiennent des facteurs antinutritionnels de diverses natures.

\section{Facteurs antinutritionnels}

Ces substances, présentes en quantités variables selon les produits végétaux, peuvent intervenir à différents niveaux tels que l'appétit, la digestion des protéines et des autres nutriments, la disponibilité des acides aminés et des minéraux ou le métabolisme comme inhibiteur d'enzymes ou comme antivitamines (Kaushik, 1990 ; Francis et al., 2001; Gatlin et al., 2007). Les mécanismes d'action de la plupart d'entre elles ne sont pas connus chez les poissons. Les effets qui leur sont attri- bués découlent d'observations expérimentales ou sont déduits par analogie avec ce qui est connu chez d'autres animaux d'élevage et chez l'humain. Chez le saumon atlantique, la présence de soja dans le régime provoque fréquemment l'apparition d'entérites (Refstie et al., 2000). Il est encore difficile de savoir lequel des facteurs antinutritionnels du soja est responsable de telles inflammations. Selon les travaux de Van den Ingh et al., (1996) qui ont testé l'effet de différentes substances indésirables isolées du soja, aucune n'induit d'entérites, ce qui suggère que l'entérite résulte de l'effet conjugué de différents facteurs antinutritionnels présents dans le soja.

Parmi les facteurs antinutritionnels les plus courants, on peut citer les facteurs antitrypsiques, inhibiteurs de protéases, l'acide phytique qui complexe le phosphore et le rend indisponible, des phyto-estrogènes (daidzéine et génistéine) qui peuvent éventuellement perturber la reproduction des poissons, des alcaloïdes qui affectent l'appétit, des glucosinolates, dont les métabolites affectent l'appétit et perturbent la synthèse des hormones thyroïdiennes, des lectines ou hémagglutinines, glycoprotéines qui se lient aux glucides, interfèrent avec l'absorption des nutriments et provoquent des phénomènes inflammatoires, des composés phénoliques comme le gossypol ou les tannins qui inhibent les enzymes digestives, des oligosaccharides tels que le raffinose et le stachyose non digestibles en raison du manque d'alphaglucosidase dans le tube digestif des poissons. Les poissons sont généralement plus sensibles à ces substances que les animaux terrestres et certaines espèces de poissons plus sensibles que d'autres. Les aliments aquacoles étant plus riches en protéines que ceux des autres animaux d'élevage, la réduction ou l'élimination de ces facteurs est de toute première importance pour que les matières premières végétales soient efficaces comme ingrédients dans les aliments piscicoles. Lorsque les facteurs antinutritionnels sont présents dans l'enveloppe des graines, le dépelliculage permet d'en éliminer une partie. Les facteurs antinutritionnels de nature protéique comme les facteurs antitrypsiques ou les lectines sont détruits par des traitements thermiques et/ou par les procédés d'extrusion. Des progrès notables ont été réalisés par la génétique végétale en sélectionnant des variétés à faible teneur en facteurs indésirables (tableau 3). Cependant, certains composés aux pro- 
Tableau 3. Caractéristiques de tourteaux d'oléagineux et de protéagineux, utilisables comme des sources protéiques dans l'alimentation des poissons.

Table 3. Main features of oil seed meals and grains available as alternative protein sources for aquafeeds.

\begin{tabular}{|c|c|c|c|c|}
\hline Produits végétaux & $\begin{array}{c}\text { Teneur en } \\
\text { protéines (\%) }\end{array}$ & AAl limitants & Substances indésirables & Commentaires \\
\hline \multicolumn{5}{|l|}{ Oléagineux } \\
\hline Tourteau de soja (Glycine max) & 45 & $\begin{array}{l}\text { Met } \\
\text { Lys } \\
\text { Thr }\end{array}$ & $\begin{array}{l}\text { Antitrypsiques } \\
\text { Phosphore phytique } \\
\text { Phyto-estrogènes } \\
\text { Oligosaccharides } \\
\text { Polysaccharides non amylacés }\end{array}$ & $\begin{array}{l}\text { Disponible sur marché } \\
\text { Profil en AAl } \\
\text { proche de la farine de poisson } \\
\text { Peu d'amidon } \\
\text { Provoque des "entérites " } \\
\text { chez le saumon }\end{array}$ \\
\hline $\begin{array}{l}\text { Tourteau de colza } \\
\text { (Brassica napus, } \\
\text { Brassica campestris) }\end{array}$ & 35 & $\begin{array}{l}\text { Lys } \\
\text { Met }\end{array}$ & $\begin{array}{l}\text { Phosphore phytique } \\
12 \% \text { fibres } \\
\text { Glucosinolates }\end{array}$ & $\begin{array}{l}\text { Nouvelles variétés } \\
\text { à faible taux de glucosinolates } \\
\text { et d'acide érucique }\end{array}$ \\
\hline $\begin{array}{l}\text { Tourteau de coton } \\
\text { (Gossypium sp.) }\end{array}$ & $40-42$ & $\begin{array}{l}\text { Lys } \\
\text { Met } \\
\text { Cys }\end{array}$ & $\begin{array}{l}\text { Gossypol } \\
\text { Teneur en fibres élevée }\end{array}$ & $\begin{array}{l}\text { Prix faible } \\
\text { Nouvelles variétés } \\
\text { à haut rendement } \\
\text { et faible teneur en gossypol }\end{array}$ \\
\hline $\begin{array}{l}\text { Tourteau de tournesol } \\
\text { (Helianthus annuus) }\end{array}$ & 36 & Lys & $\begin{array}{l}\text { Polyphénols } \\
\text { Fibres }\end{array}$ & $\begin{array}{l}\text { Peu de facteurs } \\
\text { antinutritionnels }\end{array}$ \\
\hline $\begin{array}{l}\text { Tourteau d'arachide } \\
\text { (Arachis hypogea) }\end{array}$ & $48-50$ & $\begin{array}{l}\text { Lys } \\
\text { Met }\end{array}$ & $\begin{array}{l}\text { Aflatoxine (très toxique } \\
\text { chez certaines espèces } \\
\text { comme la truite) }\end{array}$ & $\begin{array}{l}\text { Peu de facteurs } \\
\text { antinutritionnels }\end{array}$ \\
\hline \multicolumn{5}{|l|}{ Protéagineux } \\
\hline Pois (Pisum sativum) & 26 & $\begin{array}{l}\text { Lys } \\
\text { Met }\end{array}$ & $\begin{array}{l}55 \% \text { d'amidon } \\
5 \% \text { d'oligosaccharides }\end{array}$ & $\begin{array}{l}\text { Substances indésirables } \\
\text { éliminées avec la pellicule }\end{array}$ \\
\hline $\begin{array}{l}\text { Lupin doux (Lupinus albus } \\
\text { et angustifolius) }\end{array}$ & 39 & $\begin{array}{l}\text { Lys } \\
\text { Met }\end{array}$ & $\begin{array}{l}\text { Alcaloïdes } \\
8 \% \text { oligosaccharides } \\
\text { Polysaccharides non amylacés }\end{array}$ & $\begin{array}{l}\text { Peu d'amidon } \\
10 \% \text { de lipides }\end{array}$ \\
\hline
\end{tabular}

Ces données sont des valeurs moyennes indicatives en raison des variations qui interviennent en fonction des conditions de production.

LYS : Iysine ; MET : méthionine ; THR : thréonine.

priétés antinutritionnelles ne peuvent pas être éliminés, car ils sont nécessaires à la plante. C'est le cas de l'acide phytique. Il faut alors prendre des mesures pour contrecarrer leurs effets. Par exemple, pour rendre disponible le phosphore séquestré par l'acide phytique présent en grandes quantités dans les végétaux, l'addition de phytase se révèle efficace (Cheng et Hardy, 2002; Cao et al., 2007). Cette enzyme, en dégradant la liaison du phosphore phytique, augmente la biodisponibilité du phosphore et limite son rejet dans l'environnement via les excréta des poissons.

\section{Recommandations actuelles pour le remplacement de la farine de poisson par des sources protéiques végétales}

De très nombreux travaux de recherche ont été réalisés au cours des 30 dernières années pour évaluer l'intérêt et les limites de différentes matières premières végétales dans les aliments aquacoles. Dans un premier temps, les matières premières ont été testées individuellement en observant les effets de taux d'incorporation croissants sur l'ingestion, la digestion, la croissance des différentes espèces, parfois sur d'autres paramètres tels que les rejets azotés, les métabolismes, les réponses immunitaires et la qualité des produits. Ces essais ont permis de cerner le potentiel et les limites d'utilisation des sources de protéines alternatives à la farine de poisson dans l'alimentation piscicole. Un ouvrage récent (Lim et al., 2008) en présente une synthèse.

Afin de limiter les effets négatifs de chaque matière première, la stratégie adoptée a été de remplacer la farine de poisson par un mélange de sources protéiques végétales et de porter une attention particulière aux aspects identifiés comme limitants : appétence de l'aliment, digestibilité de ses composants, apport azoté, en particulier composition en acides aminés et contenu énergétique. C'est ainsi que des essais expérimentaux ont démontré la possibilité de remplacer 75 à $90 \%$ de la farine de poisson par des sources protéiques végétales dans les aliments du bar européen (Kaushik et al., 2004), de la daurade (Sitjà-Bobadilla et al., 2005), du saumon atlantique (Espe et al., 2006), de la truite arc-en-ciel (Kaushik et al., 1995 ; Palti et al., 2006). De tels taux de substitution sont encore rares dans les aliments commerciaux pour les salmonidés et les poissons marins mais ont augmenté (en moyenne 50 à $60 \%$ ) au cours des trois dernières années. Il existe déjà sur le marché des aliments dépourvus de protéines d'origine marine pour les espèces d'eau chaude comme la carpe, le tilapia, le panga qui ont des capacités supérieures aux poissons d'eau froide pour digérer les glucides apportés par les sources protéiques végétales et à utiliser le 


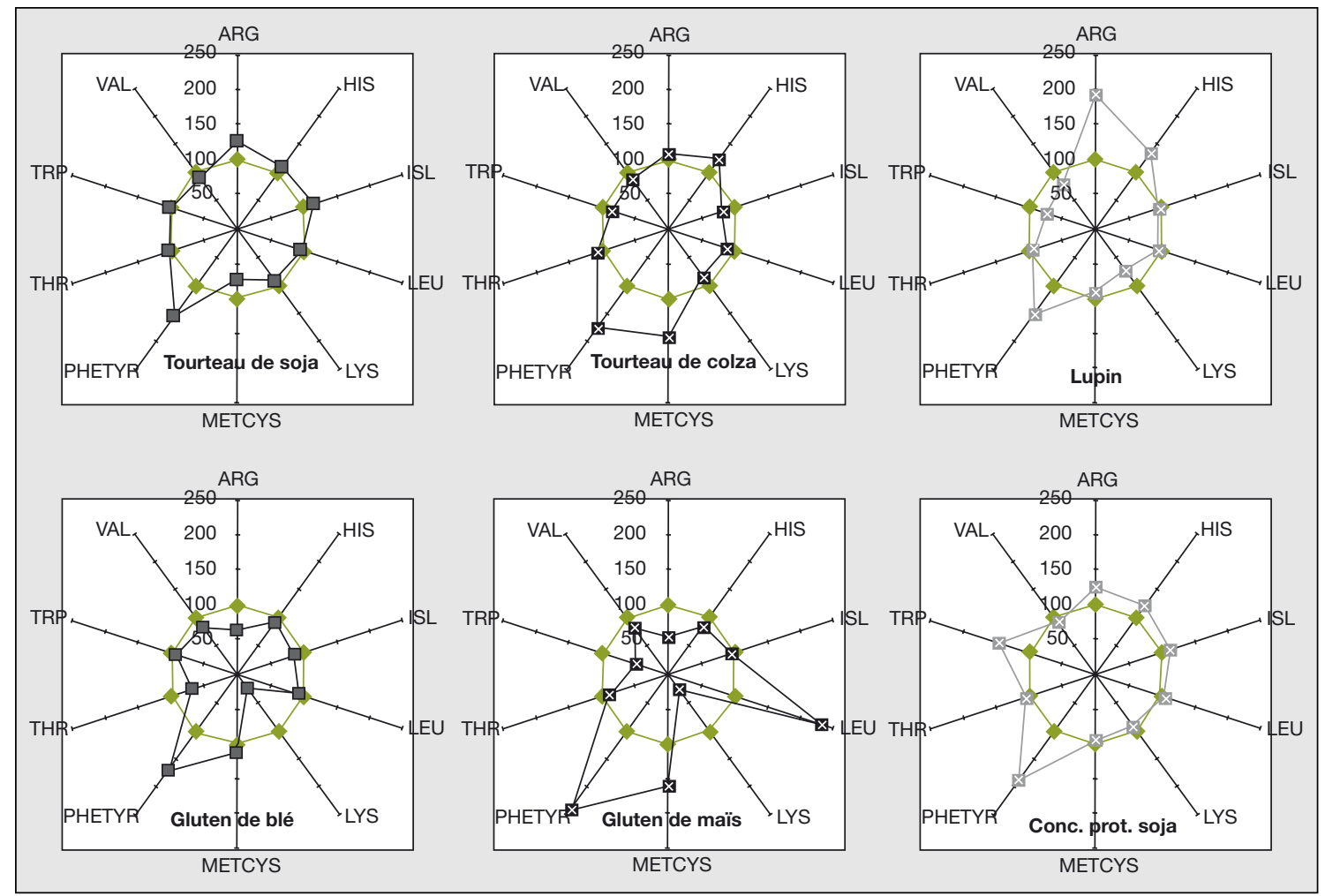

Figure 2. Profil en acides aminés indispensables de différentes sources de protéines végétales, comparé à celui de la farine de poisson.

Figure 2. Indispensable amino-acid profile of plant protein sources as compared to fish meal.

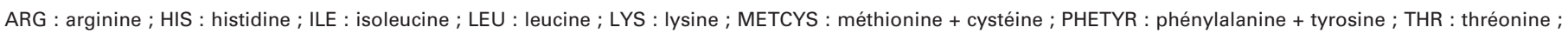
TRP : tryptophane ; VAL : valine.

glucose absorbé pour couvrir leurs besoins énergétiques (Wilson, 1994).

La présence de fortes quantités d'ingrédients végétaux dans le régime alimentaire se traduit souvent par une baisse de la consommation de l'aliment sans que l'origine de cet effet soit identifiée. Pour certaines espèces, la présence d'une faible quantité de farines de poisson pourrait suffire à maintenir une ingestion volontaire optimale. Différents facteurs d'appétence ont été étudiés: bétaïne, mélange d'acides aminés (Dias et al., 1997), farine de krill (Palti et al., 2006), hydrolysat de calmar (Espe et al., 2006) pour ne citer que les plus courants. Leur efficacité semble très variable et dépendante de l'espèce.

Les perturbations de la digestion liées à la présence d'ingrédients végétaux dans les régimes peuvent être dues à des facteurs antinutritionnels (facteurs antitrypsiques, lectines, saponines, etc.) et/ou aux polysaccharides non amylacés et aux oligosaccharides. De plus, l'amidon contenu dans les graines (céréales et protéagineux) est, à l'état natif, faiblement digéré, ce qui se traduit par une diminution de l'apport en énergie digestible. Des progrès ont été réalisés en choisissant des matières premières à faible teneur en substances indésirables et en extrudant l'aliment, ce qui conduit à la fois à inactiver certains facteurs antinutritionnels (Romarheim et al., 2005) et à améliorer la digestibilité de l'amidon (Wilson, 1994). Veiller à la digestibilité de l'ensemble des composants du régime paraît une des clés de réussite de la substitution et permet, en outre, de limiter les rejets d'origine alimentaire.

Une autre contrainte particulièrement importante à respecter est l'apport en acides aminés, qui doit couvrir les besoins des animaux. Le mélange d'ingrédients est souvent insuffisant pour répondre à ce critère, puisque, comme évoqué précédemment, pratiquement toutes les sources protéiques végétales sont déficientes en lysine et/ou méthionine. Pour mimer la composition en acides aminés de la farine de poisson, l'addition d'acides aminés est souvent nécessaire. Moyennant des précautions (enrobage, ajuste- ment du pH), il semble que les acides aminés libres apportés dans l'aliment sous forme cristalline soient utilisés aussi efficacement que les acides aminés de protéines (Rodehutscord et al., 1998 ; Espe et al., 2006).

Si les conséquences de différents taux de substitution de la farine de poisson sur la croissance des poissons ont fait l'objet de nombreuses études, peu d'entre elles ont porté sur le système immunitaire et sur la santé des poissons (Sitjà-Bobadilla et al., 2005), la reproduction (Pereira et al., 1998) et la qualité des produits (Smith et al., 1988 ; Kaushik et al., 1995 ; de Francesco et al., 2004).

Il n'apparaît pas qu'il y ait d'effets négatifs notables sur le système immunitaire et sur la qualité de la chair, même dans le cas de remplacement total. Toutefois, les pigments contenus dans certains végétaux (cas de la lutéine du maïs, par exemple) peuvent affecter la pigmentation. De même, la composition en acides gras de la chair, qui reflète celle des lipides de l'aliment, peut être modifiée. La concentration en AGPI de la série oméga 6, carac- 
téristiques des végétaux, augmente dans la chair des poissons en même temps que leur taux dans l'aliment (de Francesco et al., 2004). Cependant, ces variations sont de faible amplitude, car les sources protéiques végétales contiennent généralement peu de lipides. La teneur en AGPI oméga 3 est maintenue lorsque l'aliment contient de l'huile de poisson comme source majeure de lipides. Le remplacement de la farine de poisson par des sources végétales de protéines conduit à réduire de façon substantielle les teneurs en polluants organiques tels que dioxines et PCB-DL dans les aliments. Cependant, d'autres substances non désirées (pesticides ou herbicides) peuvent apparaître. Peu d'informations sont disponibles sur leurs conséquences biologiques. De même, peu de travaux portent sur l'emploi d'ingrédients d'origine végétale issus de plantes génétiquement modifiées dans l'alimentation des poissons.

\section{Conclusion}

Si les succès expérimentaux de remplacement total de la farine de poisson par des ingrédients végétaux sont rares (Kaushik et al., 1995; Rodehutscord et al., 1998), l'utilisation de mélanges de sources protéiques supplémentées avec des acides aminés de synthèse, dans la stricte limite des besoins des poissons, a permis de diminuer considérablement les taux de farine de poisson dans les aliments piscicoles. Cependant, des efforts de recherche sont encore nécessaires pour identifier les limites biologiques au remplacement total de la farine de poisson et pour optimiser les formules alimentaires : déterminants de l'appétit des poissons, modes d'action des facteurs antinutritionnels et des polysaccharides non amylacés, apport en minéraux et vitamines, interactions entre nutriments, conséquences sur la croissance musculaire, la reproduction, la santé et le bienêtre des poissons, ainsi que sur la qualité de la chair.

\section{Références}

Amirkolaie AK, Leenhouwers JI, Verreth YAJ, Schrama JW. Type of dietary fibre (soluble versus insoluble) influences digestion, faeces characteristics and faecal waste production in Nile tilapia (Oreochromis niloticus L.). Aquac Res 2005; 36 : 1157-66.
Cao L, Wang W, Yang C, et al. Application of microbial phytase in fish feed. Enz Microb Techno $2007 ; 40$ : 497-507.

Cheng JZ, Hardy RW. Effect of microbial phytase on apparent nutrient digestibility of barley, canola meal, wheat and wheat middling, measured in vivo using rainbow trout (Oncorhynchus mykiss). Aquac Nut 2002 ; 8 : 271-7.

Dias J, Gomes EF, Kaushik SJ. Improvement of feed intake through supplementation with an attractant mix in European seabass fed plantprotein rich diets. Aquat Living Resour 1997; $10: 385-9$.

Espe M, Lemmeb A, Petrib A, El-Mowafic A. Can atlantic salmon (Salmo salar) grow on diets devoid of fish meal? Aquaculture 2006 ; $255: 255-62$.

De Francesco M, Parisi G, Médale F, Lupi P, Kaushik S, Poli BM. Effect of long-term feeding with a plant protein mixture based diet on growth and body/fillet quality traits of large rainbow trout (Oncorhynchus mykiss). Aquaculture 2004 ; 236 : 413-29.

FAO. State of world aquaculture 2006. FAO Fisheries Technical paper, vol. 500. Rome: FAO, 2006.

Francis G, Makker HPS, Becker K. Antinutritional factors present in plant derived alternate fish feed ingredients and their effects in fish. Aquaculture 2001 ; 199 : 197-227.

Gatlin III DM, Barrows RT, Brown P, et al. Expanding the utilization of sustainable plant products in aquafeeds - a review. Aquac Res $2007 ; 38: 551-79$.

Goda AM, El-Haroun ER, Kabir Chowdhury MA. Effect of totally or partially replacing fish meal by alternative protein sources on growth of African catfish Clarias gariepinus (Burchell, 1822) reared in concrete tanks. Aquac Res 2007 ; 38 : 279-87.

Green JA, Hardy RW, Brannon EL. The optimum dietary essential: non-essential aminoacid ratio for rainbow trout (Oncorhunchus mykiss), which maximizes nitrogen retention and minimizes nitrogen excretion. Fish Physiol Biochem 2002 ; 27 : 109-15.

Kaushik SJ. Use of alternative protein sources for the intensive rearing of carnivorous fishes. In : Flos R, Tort L, Torres P, eds. Mediterranean Aquaculture. Chichester (Angleterre): Ellis Horwood, 1990.

Kaushik SJ, Cravedi JP, Lalles JP, Sumpter J, Fauconneau B, Laroche M. Partial or total replacement of fish meal by soybean protein on growth, protein utilization, potential estrogenic effects, cholesterolemia and flesh quality in rainbow trout. Aquaculture 1995; 133: 257-74.

Kaushik SJ, Coves D, Dutto G, Blanc D. Almost total replacement of fishmeal by plant protein sources in the diets for European seabass (Dicentrarchus labrax). Aquaculture 2004 ; $230: 391-404$

Krogdahl A, Hemre G-I, Mommsen TP. Carbohydrates in fish nutrition: digestion and absorption in postlarval stages. Aquac Nutr $2005 ; 11: 103-22$

Lim C, Webster CD, Lee CS. Alternative protein sources in aquaculture diets. New York: The Haworth Press ; Taylor \& Francis group, 2008.

Mambrini M, Kaushik SJ. Indispensable amino-acid requirements of fish: correspondence between quantitative data and aminoacid profiles of tissue proteins. J Appl Ichthylo $1995 ; 11: 240-7$
Médale F, Boujard T, Vallée F, et al. Voluntary feed intake, nitrogen and phophorus losses in rainbow trout (Oncorhynchus mykiss) fed increasing dietary levels of soy protein concentrate. Aquat Living Resour 1998; 11 : 239-46.

Moren M, Malde MK, Olsen RE, et al. Fluorine accumulation in atlantic salmon (Salmo salar) Atlantic cod (Gadus morhua), rainbow trout (Oncorhynchus mykiss) and Atlantic halibut (Hippoglossus hippoglossus) fed diets with krill or amphipod meals and fish meal based diets with sodium fluride (NaF) incluson. Aquaculture 2007 ; 261 : 174-81.

NRC, National Research Council. Nutrient requirements of fish. Washington (DC) : National Academy of Sciences, 1993.

Olvera-Novoa MA, Dominguez-Cen LJ, Olivera-Castillo L, Martinez-Palacios CA. Effect of the use of the micro-alga Spirulina as fish meal replacement in diets for tilapia, Oreochromis mossambicus (Peters), fry. Aquac Res $1998 ; 29: 10-7$

Palti Y, Silverstein JT, Wieman H, Philips JG, Barrows FT, Parsons JE. Evaluation of family growth response to fishmeal and glutenbased diets in rainbow trout (Oncorhyn chus mykiss). Aquaculture 2006 ; 255 : 548-56.

Pereira JOB, Reis-Henriques MA, Sanchez JL, Costa JM. Effect of protein source on the reproductive performance of female rainbow trout, Oncorhynchus mykiss (Walbaum). Aquac Res 1998; 29 : 751-60.

Refstie S, Svihus B, Shearer KD, Storebakken T. Nutrient digestibility in Atlantic salmon and broiler chickens related to viscosity and non-starch polysaccharide content in different soyabean products. Anim Feed Sci Technol $1999 ; 79: 331-45$.

Refstie S, Korsoen OJ, Storebakken T, Baeverfjord G, Lein I, Roem AJ. Differing nutritional responses to dietary soybean meal in rainbow trout (Oncorhynchus mykiss) and atlantic salmon (Salmo salar). Aquaculture 2000; 190 : 49-63.

Rodehutscord M, Borchert F, Gregus Z, Pack $M$, Pfeffer E. Free amino-acids can replace protein-bound amino-acids in test diets for studies in rainbow trout (Oncorhynchus mykiss). Aquaculture 1998; 187 : 163-76.

Romarheim $\mathrm{OH}$, Aslaksen MA, Storebakken $\mathrm{T}$ et al. Effect of extrusion on trypsin inhibitor activity and nutrient digestibility of diets based on fish meal, soybean meal and white flakes. Arch Anim Nut 2005 ; 59 : 365-75.

Shepherd CJ, Pike IH, Barlow SM. Sustainable feed resources of marine origin. Eur Aquac Soc $2005 ; 35: 59-66$.

Sitjà-Bobadilla A, Pena-Llopis S, GomezRequeni $P$, Médale $F$, Kaushik $S$, PerezSanchez J. Effect of fish meal replacement by plant protein sources on non-specific defence mechanisms and oxidative stress in gilthead sea bream (Sparus aurata). Aquaculture 2005 249 : 387-400

Smith RR, Kincaid HL, Regenstein JM, Rumsey GL. Growth, carcass composition, and taste of rainbow trout of different strains fed diets containing primarily plant or animal protein. Aquaculture 1988 ; 70 : 309-21.

Tacon AGJ, Metian M. Global overview on the use of fish meal and fish oil in industrially compounded aquafeeds: trends and future prospects. Aquaculture 2008 ; 285 : 146-58.

Tibaldi E, Kaushik SJ. Amino-acid requirements of Mediterranean fish species. Cah Options Mediterr 2005 ; 63 : 59-65. 
Van den Ingh TSGAM, Olli JJ, Krogdahl A. Alcohol-soluble components in soybeans cause morphological changes in the distal intestine of Atlantic salmon, Salmo salar L. J Fish Dis $1996 ; 19$ : 47-53.

Watanabe T. Strategies for further develpment of aquatic feeds. Fish Sci $2002 ; 68: 242-52$.
Watanabe T, Verakunpiriya V, Watanabe $\mathrm{K}$, Viswanath K, Satoh S. Feeding of rainbow trout with non-fish meal diets. Fish Sci 1998 ; $63: 258-66$.

Wilson RP. Utilization of dietary carbohydrate by fish. Aquaculture $1994 ; 124: 67-80$.
Yang Y, Xie S, Cui Y, et al. Effect of replacement of dietary fish meal by meat and bone meal and poultry by-product meal on growth and feed utilization of gibel carp, Carassius auratus gibelio. Aquac Nut $2004 ; 10$ : 289-94. 